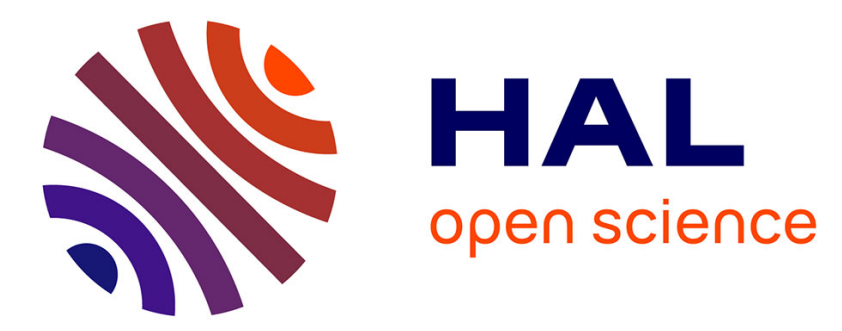

\title{
Turbulent dynamics of an incoherently pumped passive optical fiber cavity: Quasisolitons, dispersive waves, and extreme events
}

\author{
M. Conforti, Arnaud Mussot, Julien Fatome, Antonio Picozzi, Stéphane \\ Pitois, Christophe Finot, Marc Haelterman, Bertrand Kibler, Claire Michel, \\ Guy Millot
}

\section{To cite this version:}

M. Conforti, Arnaud Mussot, Julien Fatome, Antonio Picozzi, Stéphane Pitois, et al.. Turbulent dynamics of an incoherently pumped passive optical fiber cavity: Quasisolitons, dispersive waves, and extreme events. Physical Review A : Atomic, molecular, and optical physics [1990-2015], 2015, 91, pp.023823. 10.1103/PhysRevA.91.023823 . hal-01119133

\author{
HAL Id: hal-01119133 \\ https://hal.science/hal-01119133
}

Submitted on 21 Feb 2015

HAL is a multi-disciplinary open access archive for the deposit and dissemination of scientific research documents, whether they are published or not. The documents may come from teaching and research institutions in France or abroad, or from public or private research centers.
L'archive ouverte pluridisciplinaire HAL, est destinée au dépôt et à la diffusion de documents scientifiques de niveau recherche, publiés ou non, émanant des établissements d'enseignement et de recherche français ou étrangers, des laboratoires publics ou privés. 


\title{
Turbulent dynamics of an incoherently pumped passive optical fiber cavity: Quasisolitons, dispersive waves, and extreme events
}

\author{
M. Conforti, ${ }^{1}$ A. Mussot, ${ }^{1}$ J. Fatome,${ }^{2}$ A. Picozzi, ${ }^{2}$ S. Pitois,${ }^{2}$ C. Finot, ${ }^{2}$ M. Haelterman, ${ }^{3}$ B. Kibler, ${ }^{2}$ C. Michel, ${ }^{4}$ and G. Millot $^{2}$ \\ ${ }^{1}$ PhLAM/IRCICA, CNRS-Université Lille 1, UMR 8523/USR 3380, F-59655 Villeneuve d'Ascq, France \\ ${ }^{2}$ Laboratoire Interdisciplinaire Carnot de Bourgogne, UMR 6303 CNRS-Université de Bourgogne, Dijon, France \\ ${ }^{3}$ Service OPERA-photonique, Université Libre de Bruxelles, 50 Av. F.D. Roosevelt, CP 194/5, B-1050 Bruxelles, Belgique \\ ${ }^{4}$ Laboratoire de Physique de la Matière Condensée, CNRS-UMR 6622, Université de Nice Sophia Antipolis (UNSA), Nice, France
}

(Received 6 December 2014; revised manuscript received 2 February 2015; published 18 February 2015)

\begin{abstract}
We study numerically and experimentally the dynamics of an incoherently pumped passive optical fiber ring cavity nearby the zero-dispersion wavelength of the fiber. We show that the cavity exhibits a quasisoliton turbulence dynamics, whose properties are controlled by the degree of coherence of the injected pump wave: As the coherence of the pump is degraded, the cavity exhibits a transition from the quasisoliton condensation regime toward the weakly nonlinear turbulent regime characterized by short-lived rogue wave events. This behavior is reminiscent of the corresponding dynamics obtained in the purely conservative (Hamiltonian) problem. We report experimental results of an all-integrated incoherently pumped fiber cavity that provide some spectral and temporal complementary signatures of the processes predicted numerically.
\end{abstract}

DOI: 10.1103/PhysRevA.91.023823 PACS number(s): 42.65.Tg, 42.55.Wd, 42.81.Dp, 42.65.Sf

\section{INTRODUCTION}

The dynamics of turbulent waves can be characterized by the spontaneous emergence of short-lived high-amplitude waves. These rogue wave events that "appear from nowhere and disappear without a trace" [1] have been the subject of lot of studies in these last years. Aside from the hydrodynamic context [2-4], rogue waves have been recently identified in various different fields, including optical waves [5,6], capillary waves [7], superfluid helium [8], or microwaves [9]. Recent optical and hydrodynamics studies suggest that rogue wave events can be interpreted in the light of exact analytical solutions of integrable nonlinear wave equations, the socalled Akhmediev breathers [1,2,10], or more specifically their limiting cases of infinite spatial and temporal periods, the rational soliton solutions [11], such as the first-order Peregrine solution of the one-dimensional (1D) integrable nonlinear Schrödinger (NLS) equation [12]. From a more general perspective, the common feature characterizing rogue wave phenomena in the different systems is the observation of deviations from the Gaussian statistics of the wave amplitude, with long tails of the probability density function accounting for the rather frequent emission of such giant waves. We refer the reader to the following recent reviews for a detailed discussion of this vast area of research [13-16].

An important question is to understand the formation of rogue waves from a turbulent state of the wave system [3,11,17-20]. In the particular context of optics, this problem has been addressed in nonintegrable Hamiltonian systems by considering the NLS equation in the presence of third-order dispersion (TOD) $[21,22]$. This equation is known to admit quasisoliton solutions, which can be defined as radiatively decaying soliton states. More precisely, a quasisoliton is a nonlinear solution which satisfies the requirements of a classical soliton including its spatial localization, except that the structure decays very slowly during the propagation by emitting a radiation of energy far away from the structure itself [23-25]. In the problem considered here, the emission of radiation originates in the TOD term of the NLS equation. In this way, the standard soliton solution of the integrable NLS equation slowly loses its power through the emission of a radiation which is usually termed dispersive wave (DW) in the literature [26,27].

A general idea concerning the emergence of extreme events from a turbulent state is that the amount of incoherence in the system tends to prevent the formation of large-amplitude coherent wave structures in the long-term evolution of the system [19]. The total energy of the wave, i.e., the conserved Hamiltonian, plays a natural measure of the amount of incoherence in the system. By increasing the value of the Hamiltonian, different regimes were identified: the regime characterized by persistent and coherent rogue quasisoliton, then the regime in which rogue quasisolitons appear and disappear erratically, and finally the regime characterized by sporadic rogue waves events that emerge from turbulent fluctuations as bursts of light. This latter regime is characterized by a strong incoherence of the field and thus refers to the weakly nonlinear regime, which can be described by means of the wave turbulence theory [19,28,29], as discussed in detail in [30]. This quasisoliton turbulence scenario can be interpreted in analogy with standard wave condensation in the defocusing regime [19,23,31-33], in the sense that, in both cases, it is thermodynamically advantageous for the system to generate a large-scale coherent structure [a (quasi)soliton in the focusing regime or a plane wave in standard wave condensation] in order to increase the amount of disorder in the form of small-scale fluctuations. This analogy between (quasi)soliton condensation and standard wave condensation is also supported by a condensationlike curve: As the Hamiltonian increases, a transition occurs from the purely coherent and deterministic quasisoliton regime toward the fully incoherent turbulent regime [21].

This phenomenon of "quasisoliton condensation" has not yet been observed experimentally. The main reason for this can be ascribed to the presence of unavoidable linear or nonlinear (e.g., Raman-type) losses during the propagation of the random 
wave, which prevent the establishment of quasiequilibrium states in the long-term evolution of the system. Our aim in this article is to discuss, both numerically and experimentally, this phenomenon of quasisoliton turbulence in an optical fiber ring cavity. We remark in this respect that optical cavities have been considered in different circumstances to unveil interesting analogies with the phenomenon of optical wave condensation (see, e.g., Raman fiber lasers [34-36], mode-locked lasers [37], or random lasers [38], which also raised important questions such as the relation between lasing and Bose-Einstein condensation of photons [39]). In this regard, the thermalization and the Bose-Einstein condensation of a photon gas have been also reported in an optical microcavity [40].

From a different perspective, the condensation and the thermalization of classical optical waves have been also predicted to occur in an incoherently pumped passive optical cavity [41]. In this work, the turbulent dynamics of a twodimensional (2D) spatial cavity filled with a defocusing nonlinear Kerr material was studied theoretically and numerically. The analysis revealed that a very high finesse of the passive cavity is required in order to achieve wave thermalization and condensation. To increase the cavity finesse, we consider in this article a fully integrated configuration of the optical fiber ring cavity. Specifically, we consider the anomalous dispersion regime ("focusing" regime) to study quasisoliton turbulence in the temporal domain. The passive cavity is pumped by an incoherent optical wave, whose time correlation $t_{c}$ is much smaller than the round-trip time $t_{R}$. In this way, the optical beams from different cycles are mutually incoherent with each others, which makes the optical cavity "nonresonant." In this incoherent regime, the cavity does not exhibit the widely studied dynamics of pattern formation [42-46]. Instead, the dynamics of the cavity exhibits a turbulent behavior which is reminiscent of the turbulent dynamics of the purely Hamiltonian wave system considered in Ref. [21]. In this configuration, the degree of coherence of the injected pump wave in the cavity plays a role analogous to the role of the Hamiltonian in the purely conservative problem. It remarkably turns out that, as the coherence of the pump is degraded, the cavity exhibits a transition from the quasisoliton regime to the weakly nonlinear turbulent regime. Because of the limited finesse that has been reached in the experiment, the turbulent dynamics of the incoherently pumped cavity exhibits some distinguished features with respect to the conservative Hamiltonian system. Here, we discuss in detail these aspects and report experimental results that provide some spectral and temporal complementary signatures of the processes predicted numerically.

\section{MODEL}

We study the temporal dynamics of a partially coherent wave that circulates in a passive optical fiber ring cavity in the context of quasisoliton turbulence. To do so, the wavelength of the incoherent wave has been chosen to lie in the low anomalous dispersion regime of the fiber, $\beta_{2}<0$, very close to the zero-dispersion wavelength (ZDW) of the fiber. Consequently, it is necessary to account for the contribution of TOD in the NLS equation to accurately describe light propagation. The governing equation then reads as

$$
i \partial_{z} A=\frac{\beta_{2}}{2} \frac{\partial^{2} A}{\partial t^{2}}+i \frac{\beta_{3}}{6} \frac{\partial^{3} A}{\partial t^{3}}-\gamma|A|^{2} A-i \alpha A,
$$

where $z(0 \leqslant z \leqslant L)$ denotes the longitudinal spatial coordinate, $L$ being the fiber length of the cavity. $\gamma$ is the nonlinear Kerr coefficient, $\alpha$ the propagation losses, and $\beta_{2,3}$ refer to second- and third-order dispersion coefficients of the fiber [47]. We remind that in the conservative limit $(\alpha=0)$, the NLS equation (1) conserves two important quantities, the power of the optical field $P=\int|A|^{2} d t$ and the total energy (Hamiltonian) $H=E+U$, which has a linear contribution $E=\int k(\omega)|\tilde{A}|^{2}(\omega, z) d \omega$, where $k(\omega)=-\frac{\beta_{2}}{2} \omega^{2}-\frac{\beta_{3}}{6} \omega^{3}$ is the dispersion relation $[\tilde{A}(\omega, z)$ being the Fourier transform of $A(t, z)]$, and a nonlinear contribution $U=\frac{\gamma}{2} \int|A|^{4}(t, z) d t$. In the cavity configuration, the power and the Hamiltonian are no longer conserved quantities, although these quantities will be shown to reach some quasistationary values once the cavity reaches a statistically stationary regime. In the following, it proves convenient to analyze separately the contributions of second- and third-order dispersion to the linear energy $E=E_{2}+E_{3}$.

In the numerical simulations, the cavity is pumped by an incoherent optical wave. We denote by $F_{m}(t)$ the amplitude of the pump wave injected at the time $t=m t_{R}$, where $m$ is the number of round trips. As discussed above, the time correlation of the incoherent pump is much smaller than the round trip time $t_{c} \ll t_{R}$, i.e., the longitudinal coherence length is much smaller than the cavity length. In this way, the passive cavity does not behave as a resonant "phase-sensitive interferometer" [42-46], so that the temporal modes of the cavity do not play any key role in the dynamics of the incoherent wave $[41,48]$. The wave circulating in the cavity and the pump wave are thus mutually incoherent with each others, and the boundary conditions are not sensitive to the random relative phase among them:

$$
A_{m+1}(z=0, t)=\sqrt{\rho} A_{m}(z=L, t)+\sqrt{\theta} F_{m}(t),
$$

where $A_{m}(z, t)$ denotes the intracavity optical field after $m$ round trips $(0 \leqslant z \leqslant L)$, while $\rho$ and $\theta$, respectively, refer to the reflection and transmission coefficients of the field intensity $\rho+\theta=1$. Since the time correlation $\left(t_{c}\right)$ of the pump is much smaller than $t_{R}$, the pump beam $F_{m}(t)$ is uncorrelated with itself at each round trip, $\left\langle F_{m}(t) F_{p}^{*}(t)\right\rangle=\delta_{m, p}^{K} P_{F}$, where $P_{F}$ is the average power of the pump field and $\delta_{m, p}^{K}$ denotes the Kronecker symbol. We also assume that the fluctuations of the incoherent pump are statistically stationary in time, i.e., its spectrum is characterized by uncorrelated random spectral phases and the average pump power $P_{F}$ does not depend on $t$. The time correlation of the incoherent pump determines the amount of kinetic energy $E_{F}=\int \omega^{2}\left\langle|\tilde{F}|^{2}(\omega)\right\rangle d \omega$. This parameter will be shown to play a key role in the study of cavity quasisoliton turbulence. Another fundamental parameter is the finesse of the cavity $\mathcal{F}=2 \pi / \Gamma$, where $\Gamma=\theta+2 \alpha L$ denotes the effective amount of losses per round trip. Intuitively, the cavity finesse is related to the the "average lifetime that a photon spends in the cavity" $(1 / \Gamma$ being the corresponding average number of cavity round trips) or, equivalently, to the time required to fill the cavity, the so-called injection time $t_{\text {inj }}=t_{R} / \Gamma$. Once the cavity is filled, the average power 
of the intracavity optical wave reaches the stationary value $P^{s t}=P_{F} \theta / \Gamma[41]$.

\section{NUMERICAL SIMULATIONS}

We performed numerical simulations of the incoherently pumped passive cavity by integrating the NLS equation (1) for the field $A_{m}(z, t)$ from $z=0$ to $L$. The field $A_{m+1}(z=0, t)$ is then computed by applying the boundary conditions given by the cavity map (2) at each round trip. Note that, as usual, a slow-time variable can also be introduced in units of the round-trip time $T=m t_{R}$. For convenience, we normalized the problem with respect to the pump power $P_{F}$, the longitudinal nonlinear length $L_{0}=1 /\left(\gamma P_{F}\right)$, and the "healing time" $\tau_{0}=\sqrt{\left|\beta_{2}\right| L_{0}}$ [19]. The dimensionless variables are obtained through the transformations $z / L_{0} \rightarrow z ; A / \sqrt{P_{F}} \rightarrow$ $A ; F / \sqrt{P_{F}} \rightarrow F ; \alpha L_{0} \rightarrow \alpha ; L / L_{0} \rightarrow L ; t / \tau_{0} \rightarrow t ;$ and $T / \tau_{0} \rightarrow T$.

\section{A. High-finesse configuration}

In the following, we present results of the numerical simulations within two different configurations of the passive optical cavity, namely, a high-finesse and a low-finesse configuration. A remarkable result is that, contrarily to the $2 \mathrm{D}$ defocusing cavity whose condensation process was shown to require a very high finesse [41], here, the process of quasisoliton condensation has been shown to occur even with a moderate finesse of the cavity. A simple phenomenological explanation of this fact will be discussed later.

We report in Fig. 1 the numerical results realized in the case of a high-finesse cavity $\mathcal{F} \simeq 500$. This configuration typically corresponds to a pump power $P_{F}=250 \mathrm{~mW}$ injected in a fiber of length $L=6 L_{0}, L_{0}=2.95 \mathrm{~km}$, with $\beta_{2}=-6.68 \times$ $10^{-28} \mathrm{~s}^{2} / \mathrm{m}, \beta_{3}=1.15 \times 10^{-40} \mathrm{~s}^{3} / \mathrm{m}$. The absorption parameter $\alpha=1.4 \times 10^{-7} \mathrm{~m}^{-1}$ and transmission coefficients $\theta=$ 0.01 have been chosen deliberately small so as to increase the finesse of the cavity. The initial condition in the simulation is an empty cavity which is exponentially filled by the incoherent pump, with the time scale $\tau_{\text {inj }}=t_{R} / \Gamma$ [41]. Once the cavity is filled, the system reaches a statistically stationary regime, as revealed by the evolution of the spectrum of the wave as a function of the round-trip number $(m)$ reported in Figs. 1(b) and 1 (c), corresponding to low and high kinetic energy values, respectively.

\section{Role of pump incoherence on "quasisoliton condensation"}

The fundamental aspect to notice is that the properties of this statistically stationary regime strongly depend on the degree of coherence of the injected pump wave, i.e., its kinetic energy $E_{F}$. For relatively small values of $E_{F}$ (typically $E_{F}<10$, see Fig. 1), quasisolitons coherent structures are spontaneously generated, and then decay through the emission of DWs, a feature which is clearly visible through the analysis of the spectral evolution [Fig. 1(b)]. However, as the coherence of the pump wave is degraded ( $E_{F}$ is increased), the system is no longer able to efficiently generate coherent quasisoliton structures. In a loose sense, the system becomes "too hot" to generate a coherent structure (note that a similar effect in which the generation of coherent solitons was hindered by wave
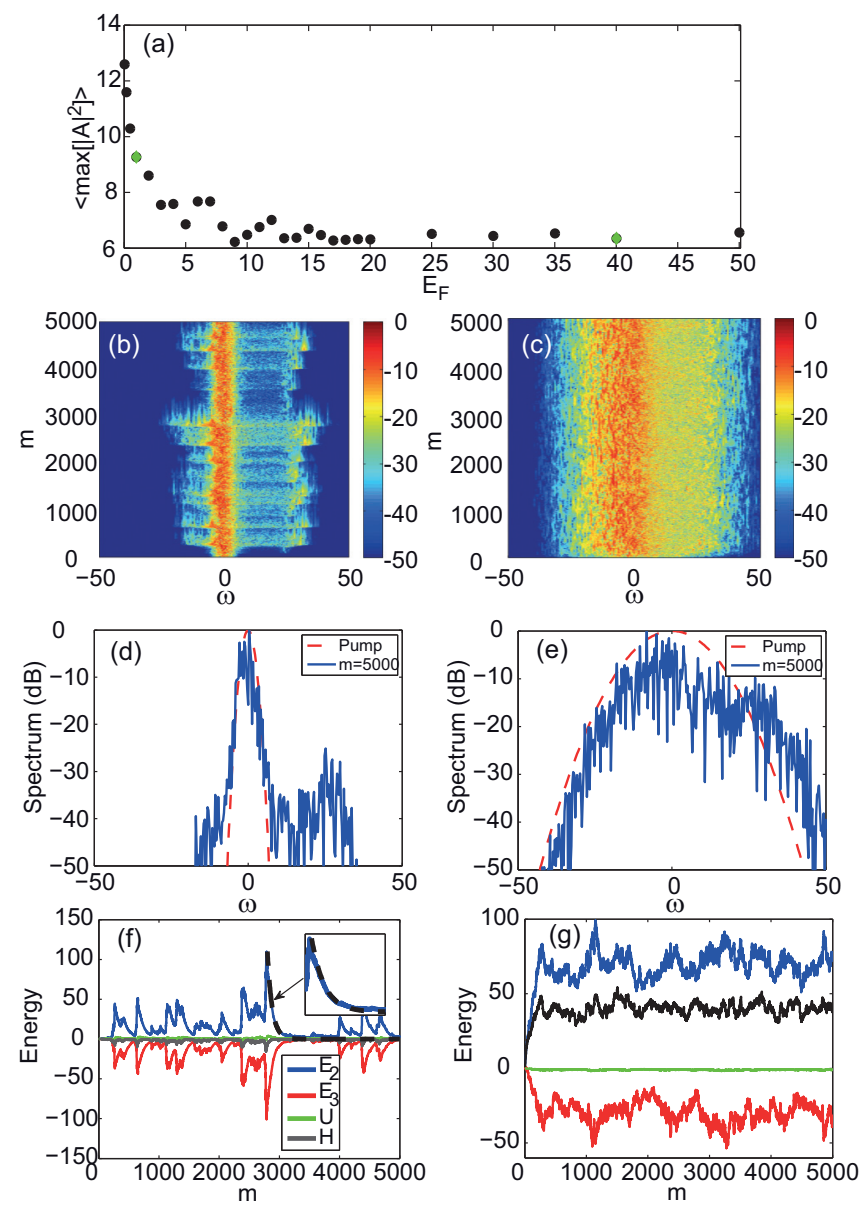

FIG. 1. (Color online) Numerical simulations of the incoherently pumped passive optical cavity in the high-finesse regime $\mathcal{F} \simeq 500$. (a) "Quasisoliton condensation curve" reporting the intensity maxima of the optical wave vs kinetic energy of the pump $E_{F}$ once a statistically stationary regime has been reached in the cavity. The left and right columns refer to the points (green, $\diamond$ ) reported in (a) for $E_{F}=1$ and $E_{F}=40$, respectively. (b), (c) Evolutions of the optical wave spectrum vs number of round trips $m$ corresponding to $E_{F}=1$ (b), $E_{F}=40$ (c), and corresponding spectral profiles recorded at $m=5000$ (d), (e). (f), (g) Evolutions of the contributions to the total energy (nonconserved Hamiltonian) $\bar{H}(T)=\bar{E}_{2}+\bar{E}_{3}+\bar{U}$ vs the slow-time $T\left(=m t_{R}\right)$, where $\bar{E}_{2}(T)$ and $\bar{E}_{3}(T)$ refer to the secondand third-order dispersion contributions to the kinetic energy $\bar{E}$. The dashed dark line in (f) denotes $\sim \exp \left(-t / \tau_{\text {inj }}\right)=\exp (-m \Gamma)$ : The effective quasisoliton lifetimes in the cavity are mainly determined by the injection time (see a zoom in the inset). Parameters are given in the text ( $\tau_{0}=1.4 \mathrm{ps}, \bar{E}_{2,3}=E_{2,3} / T_{0}, \bar{U}=U / T_{0}$ where $T_{0}$ denotes the size of the numerical temporal window $T_{0}=20 \tau_{0}$ ).

incoherence was pointed out in the context of supercontinuum generation [49]).

As discussed above in the Introduction section, this phenomenon signals the existence of a transition that can be interpreted in analogy with wave condensation. Let us briefly comment this analogy. Standard wave condensation is driven by the natural relaxation toward the thermodynamic Rayleigh-Jeans equilibrium spectrum [19,32]. The analogy between such wave condensation in the defocusing regime and (quasi)soliton condensation in the focusing regime can be 
drawn by remarking that, in both cases, the formation of a large-scale coherent structure results from the thermalization of the wave system, i.e., the natural tendency for the system to increase the amount of disorder. In other terms, by generating a large-scale coherent structure [a plane wave in the standard defocusing regime, or a (quasi)soliton in the focusing regime], the system can increase the amount of disorder in the form of small-scale fluctuations. In our cavity system, this analogy with wave condensation becomes more apparent through the analysis of the maxima of the optical wave intensity for different values of $E_{F}$, keeping constant all other parameters, in particular the pump power $P_{F}$. The results are reported in Fig. 1(a). We see that the average maximum of the quasisoliton amplitude is divided by a factor 2 for kinetic energy values larger than 10. As the kinetic energy of the pump is increased, quasisoliton structures are no longer generated, a feature which is confirmed by the spectral evolution of the incoherent wave reported in Fig. 1(c), whose spectral DW signature becomes irrelevant.

\section{Analysis of the energy contributions and the PDFs}

These observations are corroborated by the analysis of the energy contributions to the total Hamiltonian $H(T)=E_{2}+$ $E_{3}+U$. Although the Hamiltonian is no longer a conserved quantity in the cavity configuration, it tends to reach some average constant value in the statistically stationary regime of the cavity dynamics. Because of the underlying process of DW emission, the formation of a quasisoliton structure manifests itself by means of large-amplitude spikes in the evolutions of the second- and third-order contributions to the kinetic energy $E_{2}$ and $E_{3}$. It is interesting to note that the pronounced spikes in the evolutions of $E_{2}$ are almost completely compensated by those of $E_{3}$, while the nonlinear energy contribution $U$ is, in comparison, only slightly affected by the formation of quasisoliton states, as illustrated in Fig. 1(f). This analysis also reveals that robust and persistent quasisoliton states are only generated for small values of pump incoherence ( $E_{F}$ typically small than 10$)$, and in this regime their effective lifetimes in the cavity is essentially determined by the injection time $\tau_{\text {inj. This remarkable feature has been }}$ observed for different values of the finesse of the cavity [see the inset in Fig. 1(f)]. In this quasisoliton turbulence regime, the corresponding probability density function (PDF) of the intensity exhibits a strong deviation from Gaussian statistics $\left[f_{I}(I)=\exp (-I)\right.$ in normalized units], as evidenced in Fig. 2(a). Clearly, such a strong deviation is simply due to the existence of permanent quasisoliton states, as illustrated by the corresponding spatiotemporal intensity pattern reported in Fig. 2(c). The persistence of the quasisoliton structures is also corroborated by the PDF of the maxima of the intensity, which exhibits an approximately symmetric Gaussian-type shape [21,31], as illustrated by the inset of Fig. 2(a).

As the amount of pump incoherence $E_{F}$ is increased, the processes of collisions and merging of quasisolitons become strongly affected by the presence of large-amplitude turbulent fluctuations, so that quasisolitons can be rapidly generated or destroyed in an apparent random fashion. By further increasing the pump incoherence $E_{F}$, the generation of robust quasisoliton is essentially inhibited by the presence of
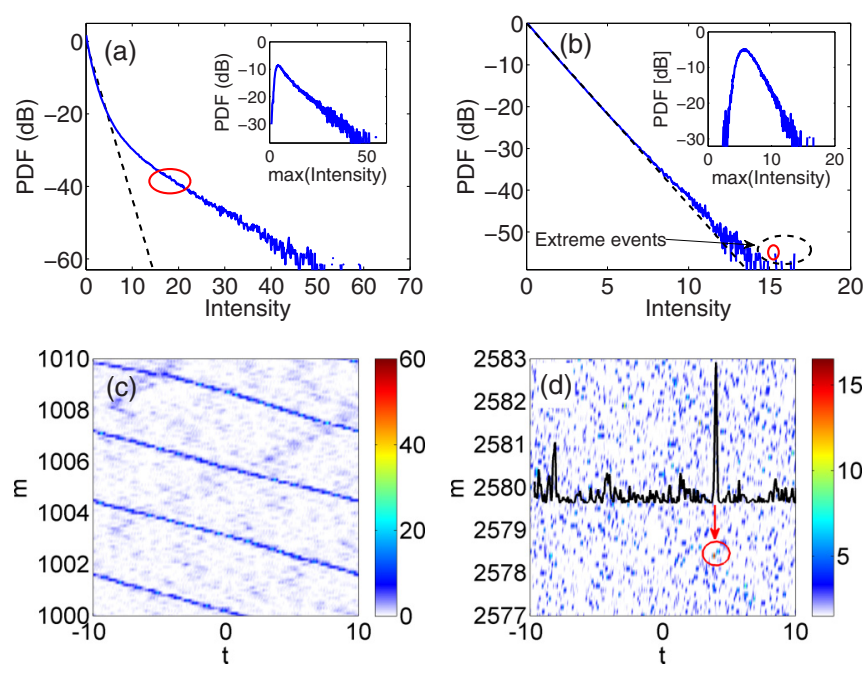

FIG. 2. (Color online) (a), (b) Intensity PDFs corresponding to a pump kinetic energy $E_{F}=1$ (a), and $E_{F}=30$ (b) [see the condensation curve in Fig. 1(a)]. The insets show the corresponding PDFs of the maxima of the intensities. (c), (d) Corresponding spacetime intensity patterns: (c) For $E_{F}=1$ the cavity field self-organizes into robust and persistent quasisoliton structures [whose typical intensity is denoted by a red circle in (a)] that propagate in the midst of small-scale fluctuations; (d) for $E_{F}=30$ the cavity field evolves in the weakly nonlinear regime and exhibits short-lived extreme events [see temporal intensity profile in (d), dark line] whose amplitudes deviate from Gaussian statistics [see red circles in (d) and (b)]. Parameters of the simulations are the same as those used in Fig. 1: $\tau_{0}=1.4$ ps, size of the temporal numerical window $T_{0}=20 \tau_{0}$, the vertical coordinate $m$ in (c) and (d) denotes the number of round trips (see Fig. 1).

large-amplitude turbulent fluctuations. This refers to the weakly nonlinear turbulent regime, in which linear dispersive effects dominate nonlinear effects, as illustrated by the comparison of linear and nonlinear contributions to the total energy [see Fig. 1(g), $\left|E_{2,3}\right| \gg|U|$ ] [19]. This regime corresponds to the tail of the quasisoliton "condensation curve" ( $E_{F}$ typically greater than 20), which is characterized by shortlived extreme waves events that emerge from the fluctuations in a sporadic way. This is illustrated by the intensity PDF in Fig. 2(b), which reflects Gaussian statistics, except for the presence of rare large-amplitudes events. These extreme events are no longer related to robust quasisoliton structures, as clearly evidenced by the corresponding spatiotemporal intensity pattern of the field reported in Fig. 2(d). This is also illustrated by the corresponding PDF of the maxima of the intensity reported in the inset of Fig. 2(b), whose asymmetric shape denotes the nonpersistent character of the fluctuations, which exhibit large deviations and tend to favor the high-intensity tail of the maxima of the PDF. Also, note that the average of this PDF decreases for the broader spectrum [compare insets of Figs. 2(a) and 2(b)], a feature consistent with the quasisoliton "condensation curve" in Fig. 1(a).

\section{B. Low-finesse configuration}

Let us now discuss a more realistic experimental configuration characterized by a significantly reduced finesse $\mathcal{F} \simeq 22$, i.e., an average photon lifetime of $\sim 3.5$ round trips. This 

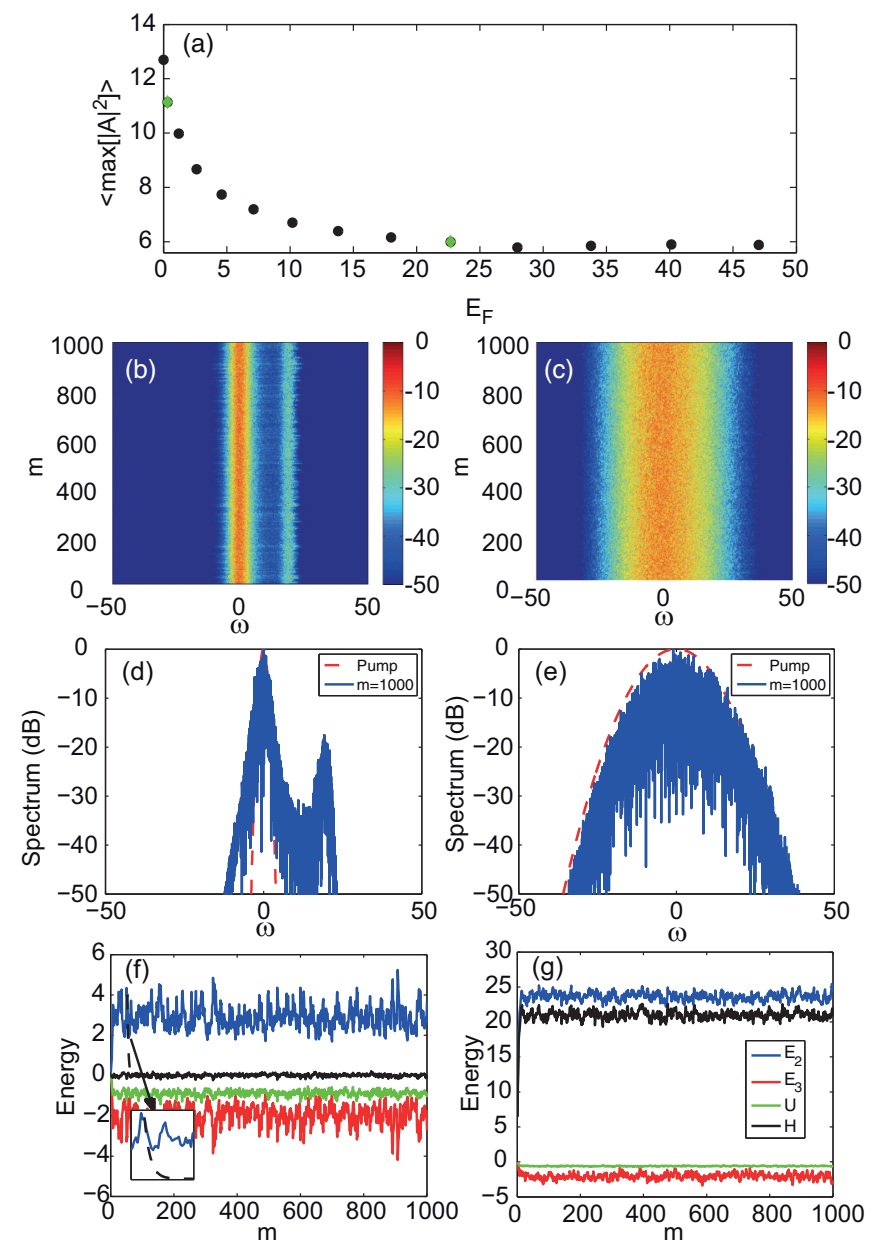

FIG. 3. (Color online) Numerical simulations of the incoherently pumped passive optical cavity in a low-finesse regime $\mathcal{F} \simeq 22$. (a) Quasisoliton condensation curve reporting the intensity maxima of the optical wave vs kinetic energy of the pump $E_{F}$ once a statistically stationary regime has been reached. (b), (c) Evolutions of the optical wave spectrum vs number of round trips $m$ corresponding to $E_{F}=0.33$ (b), $E_{F}=22.7$ (c), and corresponding spectral profiles recorded at $m=1000$ (d), (e). (f), (g) Evolutions of the contributions to the total energy (nonconserved Hamiltonian) $\bar{H}=\bar{E}_{2}+\bar{E}_{3}+\bar{U}$ vs the slow time $T\left(=m t_{R}\right)$, where $\bar{E}_{2}(T)$ and $\bar{E}_{3}(T)$ refer to the second- and third-order dispersion contributions to the kinetic energy $\bar{E}$. The green diamonds in (a) correspond to the value of $E_{F}$ used. The dashed dark line in (f) denotes $\sim \exp \left(-t / \tau_{\text {inj }}\right)=\exp (-m \Gamma)$ : the effective quasisoliton lifetimes in the cavity is mainly determined by the injection time (see a zoom in the inset). Parameters are given in the text $\left(\tau_{0}=1.19 \mathrm{ps}, \bar{E}_{2,3}=E_{2,3} / T_{0}, \bar{U}=U / T_{0}\right.$ where $T_{0}$ denotes the size of the numerical temporal window $T_{0}=320 \tau_{0}$ ).

configuration typically corresponds to the experiment that will be discussed in the next Sec. IV. Here, we briefly compare the numerical simulations of this configuration with those discussed in the high-finesse case discussed above. This lowfinesse configuration corresponds to a pump power of $P_{F}=$ $600 \mathrm{~mW}$, a fiber length $L=L_{0}, L_{0}=2.95 \mathrm{~km}$, with $\beta_{2}=$ $-4.84 \times 10^{-28} \mathrm{~s}^{2} / \mathrm{m}, \beta_{3}=1.15 \times 10^{-40} \mathrm{~s}^{3} / \mathrm{m}$. The significant reduction of the finesse is due to the choice of a more realistic absorption parameter $\alpha=4.61 \times 10^{-5} \mathrm{~m}^{-1}(=0.2 \mathrm{~dB} / \mathrm{km})$ and transmission coefficient $\theta=0.095$.
The remarkable and unexpected result is that, despite the small value of the finesse, the dynamics of the cavity exhibits properties similar to those discussed in the highfinesse configuration, as revealed by the comparison of Figs. 3 and 1(a). In particular, despite the low finesse, the cavity exhibits an efficient process of quasisoliton "condensation" [see Figs. 3(a)-1(a)]. As already commented above, this is in contrast with 2D cavity condensation in a defocusing Kerr medium, which can only occur in the presence of a very high finesse [41]. Let us briefly comment on this unexpected result. In a defocusing medium, wave condensation manifests itself by the spontaneous formation of a plane wave [29,32]. A distinguished feature with respect to quasisoliton condensation is that the formation of a plane-wave condensate requires a background of thermalized small-scale fluctuations (because wave condensation is driven by the divergence of the equilibrium Rayleigh-Jeans distribution [32]). In contrast, a (quasi)soliton is inherently a localized structure that can be generated locally in space, its robustness ensuring some long-time persistence. Accordingly, because of their localized character, quasisoliton structures are less sensitive to the boundary conditions of the system. This can provide a simple interpretation of the fact that condensation curves reported for high and low values of the finesse exhibit similar properties [see Figs. 1(a) and 3(a)].

Note, however, that, despite such similar properties of the corresponding condensation curves, the actual dynamics of the incoherent wave is significantly affected by the finesse of the cavity. Notice in particular that the effective lifetime of quasisolitons is significantly reduced in the low-finesse cavity case since the lifetime is essentially determined by the injection time $\tau_{\text {inj }}=t_{R} / \Gamma$, as illustrated in Fig. 3(f). As a consequence, the spectral signature associated to the emission of DWs becomes less pronounced in the low-finesse case, so that quasisoliton collisions play a minor role as compared to the high-finesse case considered in Fig. 1. Remark that the higher spectral resolution of simulations performed in Fig. 3 as compared to Fig. 1 is due to the larger temporal numerical window $T_{0}$ (see the figure captions), which has been chosen in such a way to keep almost the same ratio $T_{0} / \tau_{\text {inj }}$ in both low- and high-finesse cases. Finally, note that the PDFs of the low-finesse configuration of the intracavity optical field will be analyzed later in relation with the experimental results.

\section{EXPERIMENTAL SETUP}

The experimental setup is displayed in Fig. 4. This experimental configuration has to fulfill two stringent and nonobvious requirements. The first issue is to provide a widely tunable incoherent signal whose spectral bandwidth can be adjusted from $10 \mathrm{GHz}$ to $1 \mathrm{THz}$ without significantly altering its spectral profile. To this aim, a 23-dBm erbium-based amplified spontaneous noise emission source (ASE) centered in the C-band is first polarized. In order to increase the peak power at the amplification stage and also to trig the monitoring process at the receiver on the real-time oscilloscope, the initial signal is segmented in the time domain by means of a $\mathrm{LiNbO}_{3}$ Mach-Zehnder modulator driven by a programmable pulse pattern generator (PPG). The final temporal signal consists in a burst of $1.5 \mathrm{~ns}$ of incoherent waves at a repetition rate of 


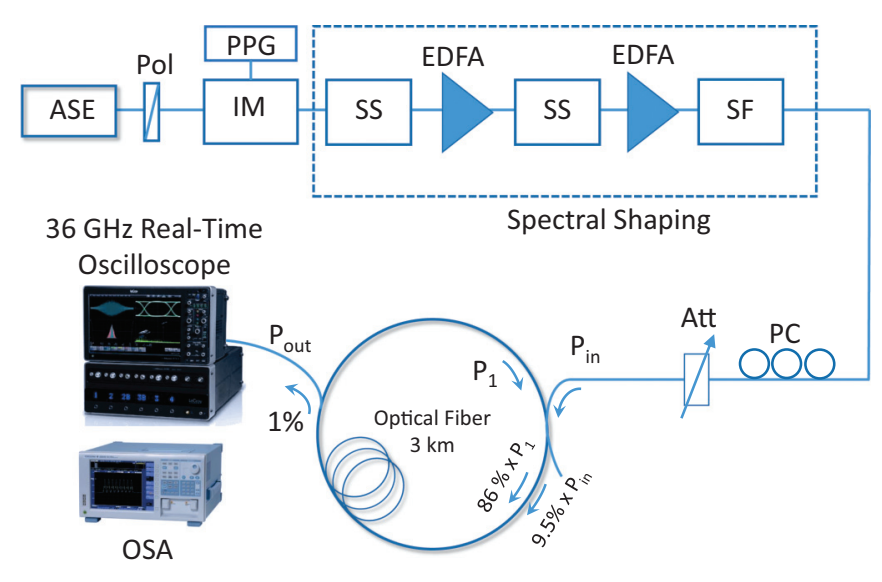

FIG. 4. (Color online) Experimental setup. ASE: amplified spontaneous emission; PPG: pulse pattern generator; Pol: polarizer; IM: intensity modulator; SS: spectral shaper; SF: spectral filter; EDFA: erbium-doped fiber amplifier; OSA: optical spectrum analyzer; Att: attenuator; PC: polarization controller.

33.3 MHz corresponding exactly to the 656th of the cavity ring harmonics, therefore enabling the temporal synchronization of the incoming energy with the incoherent recirculating burst. As discussed above, contrary to resonant cavities $[44,46]$, the incoherent nature of the wave under study does not require us to implement an active and accurate cavity length stabilization feedback loop. It is important to note that the temporal duration of the recirculating bursts as well as their temporal separation are long enough to ensure the validity of a continuously incident incoherent wave approximation. The amplification stage consists in two consecutive erbium-doped fiber amplifiers (EDFA) associated to two programmable liquid-crystal-based optical filters, which allow us to compensate for the gain flatness imperfections on more than $1 \mathrm{THz}$ of amplification band and also to remove the out-band spontaneous noise emission. A tunable bandwidth optical filter, centered on $1550 \mathrm{~nm}$, allows us to adjust the incoherent nature of the incident signal incoming into the cavity and a polarization controller was included before injection so as to optimize the polarization state of the light injected in the ring cavity.

The second challenging requirement of this experimental setup is to reduce the level of optical losses experienced over a round trip and to operate in the vicinity of the ZDW. We achieved these points by taking advantage of telecom-grade fibers and components. Specifically, the cavity is made of a $2.95-\mathrm{km}$-long dispersion shifted fiber (DSF) characterized by a Kerr coefficient $\gamma=1.7 \mathrm{~W}^{-1} \mathrm{~km}^{-1}$, a chromatic dispersion coefficient at $1550 \mathrm{~nm} \beta_{2}=-4.84 \times 10^{-28} \mathrm{~s}^{2} / \mathrm{m}$, a third-order dispersion $\beta_{3}=1.1 \times 10^{-40} \mathrm{~s}^{3} / \mathrm{m}$ and losses of $0.2 \mathrm{~dB} / \mathrm{km}$. A $10: 90$ coupler is used to both inject the incoherent wave into the system as well as to close the cavity ring. A second 1:99 coupler was inserted within the loop in order to extract and monitor the propagating signal. In order to minimize the overall losses, all the fiber components have been very carefully spliced. Taking into account the whole losses of the fiber ring, we estimate the effective lifetime of the photons circulating in the cavity to around four round trips. At the output of the system, an optical spectrum analyzer was plugged in the $1 \%$ control port of the cavity in order to characterize the recirculating signal in the spectral domain while for the temporal measurements, detection was also performed thanks to a $50-\mathrm{GHz}$ photodiode connected to a $36-\mathrm{GHz}$ bandwidth real-time oscilloscope provided by Teledine Lecroy LabMaster $10 \mathrm{Zi}$. We finally note that there are typically five orders of magnitudes between the round-trip time $t_{R}(\sim 15 \mu \mathrm{s})$ and the coherence time $t_{c}$ (which varies between $50 \mathrm{ps}$ and $500 \mathrm{fs}$ ), so that the considered cavity clearly operates in a nonresonant incoherent regime.

\section{EXPERIMENTAL RESULTS}

\section{A. Results by varying the pump wavelength}

We have first checked that the "closed fiber ring" play an important role in the incoherently pumped cavity since no spectral signature of the DW has been identified experimentally for an "open cavity," i.e., for a single passage of the incoherent pump through the fiber. To further validate the dynamical behavior of the cavity, we have studied the influence of the pump wavelength on the generation of quasisolitons by monitoring the corresponding DW spectral profile. The spectral bandwidth of the incoherent pump was fixed to $10 \mathrm{GHz}$ for an average power of $893 \mathrm{~mW}$ (the average power means here a temporal average over a temporal window much larger than the time correlation of the pump). Figure 5(a) summarizes the experimental results of the wave spectrum, which have been compared with the corresponding numerical simulations realized with the experimental parameters [see Fig. 5(b)]. For pump wavelengths slightly larger than the fiber ZDW
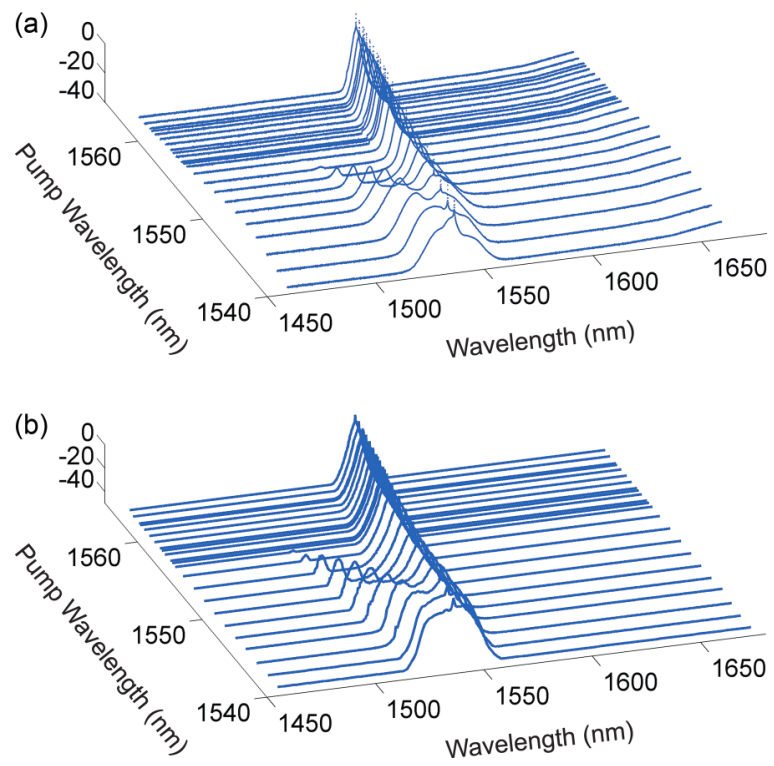

FIG. 5. (Color online) (a) Experimental spectra recorded by varying the wavelength of the incoherent pump injected in the cavity, and (b) corresponding numerical NLS simulations of Eqs. (1) and (2) realized with the experimental parameters. The average power of the incoherent pump is kept fixed to $893 \mathrm{~mW}$, while its spectral bandwidth to $10 \mathrm{GHz}$ (see the text for all experimental parameters of the incoherently pumped passive optical cavity). Note the satisfactory qualitative agreement between the experimental results and the numerical results obtained with the experimental parameters given in Sec. IV. 
(1545 nm), we can observe an efficient generation of DWs which provides an indirect spectral evidence of the presence of quasisoliton structures in the cavity. The efficiency of the generation of DWs rapidly drops as the pump wavelength is increased. The reduction of the DW spectral line is due to the fact that, by increasing the pump wavelength (i.e., by increasing the ratio $\left.\left|\beta_{2} / \beta_{3}\right|\right)$, the optical wave frequency moves away from the ZDW of the fiber, so that the generation of DWs is significantly reduced because of the increasing phase mismatch between the quasisoliton and the associated DW. We have also checked in the output spectrum of the cavity that no pump depletion occurred due to the Raman effect. This experimental study allowed us to control the dynamics of the incoherently pumped cavity. In particular, a good qualitative agreement has been obtained between the experimental results and the numerical simulations of NLS equations (1) and (2), which thus validates our model of the incoherently pumped passive cavity.

We finally note that the experimental spectra recorded here refer to averaged spectra, i.e., monitored by means of an optical spectrum analyzer. Even if new methods have recently emerged in order to record the evolution of the spectrum in real time at each round trip [6] and to experimentally reconstruct maps such as that reported in Fig. 1(b), it is worth noting that such methods cannot be implemented here because of the ns duration of the optical bursts and the very low level of the signal extracted from the cavity (a few $\mathrm{mW}$ ).

\section{B. Results by varying the pump coherence}

In a second series of measurements, we have investigated the influence of pump incoherence on the efficiency of quasisoliton generation and the associated process of DW emission. Note that, to properly study the impact of pump incoherence, the bandwidth of the incoherent pump has been varied by keeping constant its average power, as it was discussed above through the analysis of the condensation curve. Typical examples of the recorded experimental spectra are reported in Fig. 6 for two different bandwidths of the incoherent pump, $10 \mathrm{GHz}$ and $1 \mathrm{THz}$, and the same average power, $893 \mathrm{~mW}$. Note that the central wavelength of the incoherent pump is kept fixed to $1549.85 \mathrm{~nm}$. As expected from the previous numerical study reported through Figs. 1-3, the impact of pump incoherence is to reduce the efficiency of quasisoliton generation, which in turn entails a reduction of the DW spectral line. These experimental results are confirmed by the numerical simulations of the NLS equations (1) and (2) realized with the experimental parameters [Fig. 6 (red line)]. Unfortunately, we have not been able to observe the complete suppression of the DW spectral signature of quasisoliton generation predicted numerically through the quasisoliton condensation curve (Figs. 1-3) because such regime requires too broad spectral bandwidths of the injected incoherent pump [estimated around 3.5-4 THz according to Fig. 3(a)].

\section{Experimental and numerical analysis of probability density functions}

In this section, we corroborate the previous study of the spectral properties of the cavity dynamics by a study of its temporal dynamics through the analysis of the PDFs of the
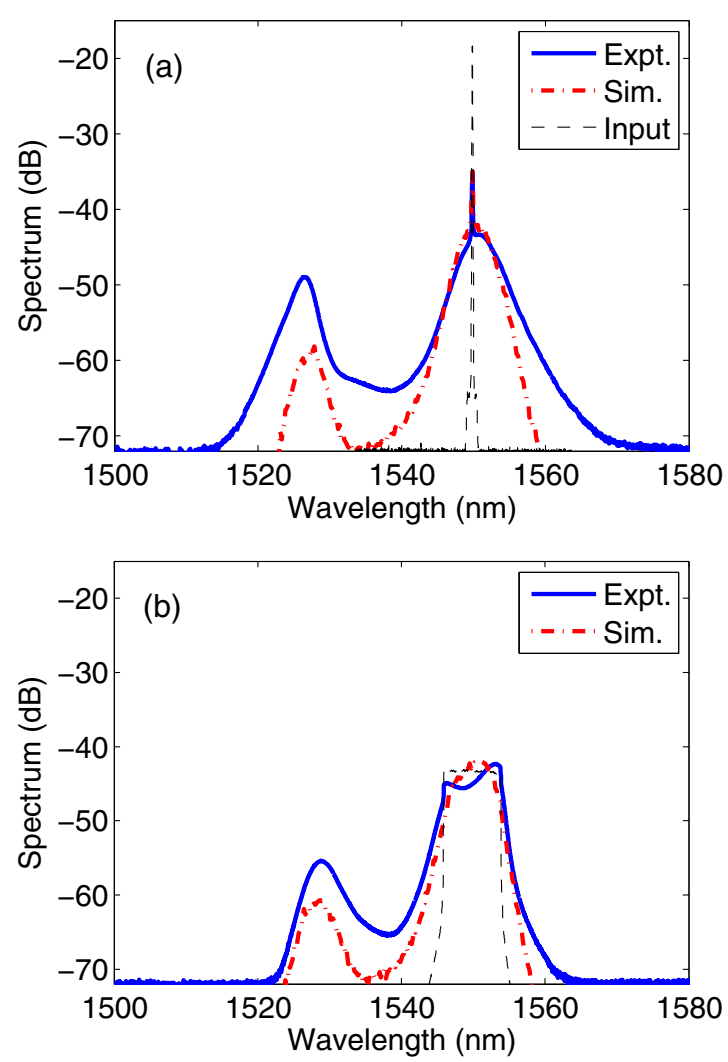

FIG. 6. (Color online) Experimental (solid blue curve) and numerical (dashed-dotted red curve) spectral profiles of the intracavity optical wave corresponding to an injected incoherent pump of spectral bandwidth $10 \mathrm{GHz}$ (a) and $1 \mathrm{THz}$ (b) for a fixed value of the pump intensity $(893 \mathrm{~mW})$ : The DW spectral signature that evidences the presence of quasisolitons is reduced as the coherence of the pump is degraded. The spectrum of the injected incoherent pump wave is reported in dashed dark line. The numerical NLS simulations of Eqs. (1) and (2) have been performed with the experimental parameters given in the text (see Sec. IV).

optical wave intensity. Considering the short-time correlation which characterizes the optical wave (ps range) and the limited bandwidth of the oscilloscope $(36 \mathrm{GHz})$, the experimental recording necessarily leads to a significant smoothing of the temporal intensity profile, which in turn significantly affects the corresponding intensity PDF. We thus start our study by discussing the properties of the PDFs computed by performing numerical simulations realized with the experimental parameters. We report in Fig. 7 the PDFs of the intensity, and of the corresponding maxima, calculated with the three different incoherent pump spectra discussed above in the experiment in Sec. V B. As discussed above through Fig. 2, the PDF of the intensity exhibits a significant deviation from Gaussian statistics, a feature which is due to the presence of quasisoliton coherent structures in the dynamics of the incoherent wave. Also note that the PDFs corresponding to pump spectral widths of 0.01 and $0.1 \mathrm{THz}$ exhibit very similar properties. This is due to the fact that the time correlation of the narrower spectrum $(0.01 \mathrm{THz})$ is much larger than the MI period $(\sim 5 \mathrm{ps})$. In this way, each individual fluctuation of the incoherent wave develops its own MI, which rapidly brings the dynamics in 

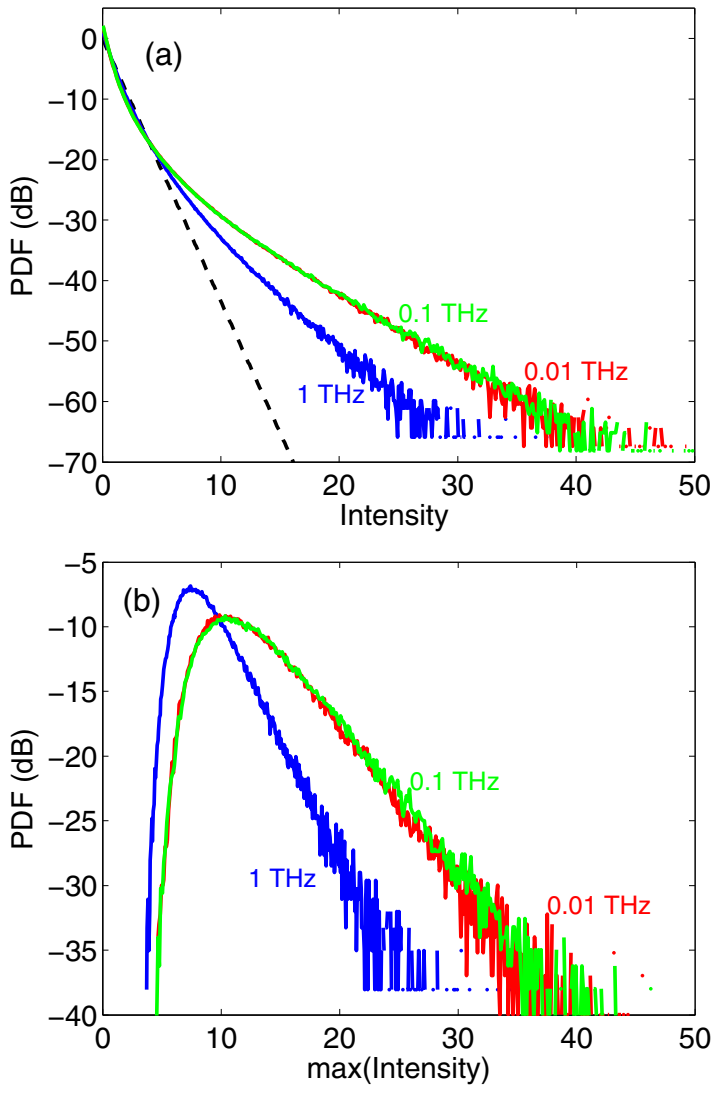

FIG. 7. (Color online) PDFs of the intensity (a), and of the maxima of the intensity (b), computed numerically by solving the NLS equations (1) and (2), with three different pumps of spectral widths $0.01 \mathrm{THz}$ (red), $0.1 \mathrm{THz}$ (green), and $1 \mathrm{THz}$ (blue) (in log scale). The dashed line in (a) stands for Gaussian statistics of the field amplitude, i.e., exponential PDF for the intensity. The incoherence of the pump wave significantly reduces the efficiency of generation of high-amplitude coherent quasisoliton structures.

a regime similar to that obtained with a pump-spectral width of $0.1 \mathrm{THz}$. On the other hand, the deviation from Gaussian statistics for these two pump waves is larger than that reported with the broader spectral width of $1 \mathrm{THz}$ (note that in this latter case, MI is essentially suppressed by pump incoherence [50]). This results from the fact that the incoherence of the broader spectral pump (1 THz) is sufficient to significantly reduce the generation of quasisolitons, as discussed in detail through the analysis of the quasisoliton condensation curve [Fig. 3(a)]. Note, however that, as discussed above through Fig. 6, a complete suppression of quasisoliton generation requires a spectral bandwidth of the incoherent pump broader than 1 $\mathrm{THz}$, which explains why there is still a significant deviation of the PDF from Gaussian statistics.

The corresponding PDFs of the maxima of the intensity are reported in Fig. 7(b). As expected from the condensation curve discussed above in Figs. 1-3, the maximum of the PDF decreases for the broader spectrum $(1 \mathrm{THz})$ as compared to the two narrow spectra $(0.01$ and $0.1 \mathrm{THz})$, simply because the broader spectrum significantly reduce the quasisoliton average amplitudes. Also notice that, as already commented through the high-finesse cavity configuration in Fig. 2, the
PDF gets asymmetric for the broader spectrum, a feature that reflects the nonpersistent character of the quasisoliton structure, whose significant fluctuations tend to favor the high intensity tail of the PDF. It is important to remark that the PDFs properties discussed here exhibit properties very similar to those discussed in the purely conservative (Hamiltonian) problem (see [21]), despite the fact that the cavity considered in these simulations corresponds to the realistic low-finesse experimental configuration.

This general discussion is confirmed by the experimental recordings of the intensity PDFs reported in Fig. 8(a). The intensity PDFs have been captured at the output of the passive cavity for the three different bandwidths of the incoherent pump (10 GHz, $100 \mathrm{GHz}$, and $1 \mathrm{THz})$ and for a constant average power of the incoherent pump $893 \mathrm{~mW}$, as well as a fixed
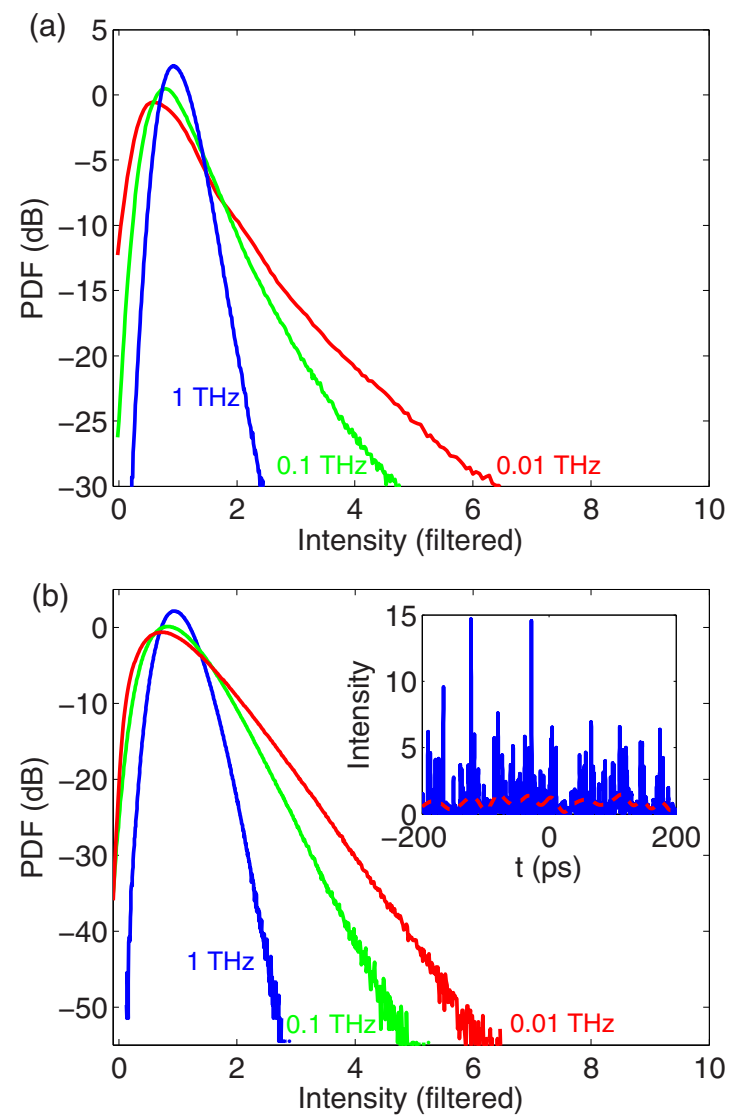

FIG. 8. (Color online) (a) PDFs of the intensity recorded experimentally with an oscilloscope of spectral bandwidth $36 \mathrm{GHz}$, for three different pumps of spectral widths $0.01 \mathrm{THz}$ (red), $0.1 \mathrm{THz}$ (green), and $1 \mathrm{THz}$ (blue), with a constant average power of the incoherent pump, $893 \mathrm{~mW}$. (b) Corresponding PDFs obtained by numerical simulations of Eqs. (1) and (2) with the experimental parameters, in which the limited spectral bandwidth of the oscilloscope has been modeled by a square-shaped (super-Gaussian, $n=8$ ) spectral filter of $36 \mathrm{GHz}$. The inset shows a typical temporal intensity profile of the NLS simulation (solid blue curve) and the corresponding smoothed profile (dashed red curve). A qualitative agreement is obtained between the numerical results and the PDFs recorded experimentally, thus confirming that the main impact of pump incoherence is to significantly reduce the efficiency of generation of quasisoliton structures. 
central pump wavelength of $1549.85 \mathrm{~nm}$. These experimental results corroborate the results anticipated by the numerical simulations in the sense that we can observe a significant reduction of the tail of the intensity PDFs as the incoherence of the pump is increased. In particular, the PDF tail corresponding to $1-\mathrm{THz}$ pump bandwidth is dramatically reduced, thus confirming that MI and the associated process of quasisoliton generation are reduced in a substantial way by pump incoherence. However, it is worth noting that there are important qualitative differences between the experimental PDFs reported in Fig. 8(a) and the corresponding numerical simulations reported in Fig. 7(a), in particular as regard the presence of the offsets in the experimental PDFs. Such important differences are due to the limited bandwidth of our real-time oscilloscope detection whose spectral bandwidth is $36 \mathrm{GHz}$. This interpretation is clearly confirmed by the numerical simulations of Eqs. (1) and (2) reported in Fig. 8(b). The PDFs reported in this figure actually refer to those reported in Fig. 7(a), except that the role of the limited bandwidth of the oscilloscope has been taken into account by means of a super-Gaussian spectral filtering process. The essential role of the limited bandwidth of the oscilloscope detection is clearly illustrated in the inset of Fig. 8(b), which shows a typical temporal intensity profile of the NLS simulation (blue) and the corresponding smoothed profile (red) due to the spectral filtering process of the oscilloscope.

The spectral filtering process due to the limited bandwidth of the detection can be modeled as a convolution in the temporal domain: The filtered intensity is related to the original intensity by $I_{f}(t)=I(t) * h(t)$, where the Fourier transform of $h(t)$ refers to the spectral bandwidth of the oscilloscope, here $36 \mathrm{GHz}$. We can discretize the convolution integral with a time step of the order of the time correlation of $I(t)$, so that $I_{f}(t)$ can be considered as a sum of independent random variables, which, according to the central limit theorem, leads to a Gaussian PDF for $I_{f}(t)$. This should merely explain why the intensity PDF for the high incoherent pump (1 THz), for which the smoothing process is the more effective, turns to be essentially Gaussian shaped by the filtering process (see blue curve in Fig. 8). We note that the impact of the limited bandwidth of laboratory equipment on the analysis of PDF properties of stochastic signals has been recently considered in Ref. [51]. In spite of this significant smoothing of large-amplitude temporal fluctuations due to the experimental detection, the experimental setup allows us to identify the impact of pump incoherence on the generation of coherent quasisoliton structures.

\section{CONCLUSION}

We have reported an experimental and numerical study of an incoherently pumped passive optical fiber ring cavity.
The dynamics of the optical cavity has been shown to be essentially characterized by a quasisoliton turbulence process. More specifically, the generation of quasisolitons is controlled by the degree of coherence of the injected pump wave: As the coherence of the pump is degraded, the system undergoes a transition from the quasisoliton turbulence regime toward the highly incoherent (weakly nonlinear) turbulent regime characterized by short-lived and large-amplitude rare events, a transition that can be interpreted in analogy with quasisoliton condensation. We have realized an all-integrated incoherently pumped passive optical cavity, whose dynamics has been characterized by means of complementary spectral and temporal PDF measurements.

A remarkable unexpected result of our study is that quasisoliton condensation can take place efficiently, even in the presence of a low finesse of the cavity, in contrast with wave condensation in $2 \mathrm{D}$ defocusing media, which was shown to require a high-cavity finesse [41]. We have interpreted this result as a consequence of the fact that the process of thermalization of an optical wave constitutes a prerequisite for the phenomenon of wave condensation in a defocusing medium, while wave thermalization is known to require a high-cavity finesse. There is another important difference which distinguishes wave condensation and (quasi)soliton condensation. Wave condensation is known to exhibit a property of long-range order and coherence [52], in the sense that the correlation function of the field amplitude does not decay at infinity, $\lim _{\left|\boldsymbol{r}-\boldsymbol{r}^{\prime}\right| \rightarrow \infty}\left\langle A(\boldsymbol{r}) A^{*}\left(\boldsymbol{r}^{\prime}\right)\right\rangle \neq 0$, a property which is consistent with the intuitive idea that, ideally, the coherence length of a plane wave diverges to infinity [32]. This is in contrast with the spatial localized character of a (quasi)soliton, which naturally limits the range of coherence to the characteristic spatial width of the (quasi)soliton structure. In other terms, wave condensation appears to be more sensitive to the "boundary conditions" of the system, and thus results less robust than (quasi)soliton condensation when considered in an optical cavity system. The understanding of this aspect will be the subject of future investigations, by also including a spatial [53] or temporal [54] nonlocal response of the nonlinearity which is known to introduce a different turbulent behavior of the system.

\section{ACKNOWLEDGMENT}

The authors acknowledge support from the French National Research Agency (Grant No. ANR-12-BS04-0011 OPTIROC, and Grant No. ANR-14-ACHN-0014-01 NoAWE), the iXCore research foundation, and the Labex ACTION (Grant No. ANR11-LABX-01-01). The authors thank P. Suret and S. Randoux for fruitful discussions.
[1] N. Akhmediev, A. Ankiewicz, and M. Taki, Phys. Lett. A 373, 675 (2009).

[2] A. Osborne, Nonlinear Ocean Waves \& the Inverse Scattering Transform (Academic, San Diego, 2010).

[3] M. Onorato, A. R. Osborne, M. Serio, and S. Bertone, Phys. Rev. Lett. 86, 5831 (2001).
[4] A. Chabchoub, N. Hoffmann, M. Onorato, A. Slunyaev, A. Sergeeva, E. Pelinovsky, and N. Akhmediev, Phys. Rev. E 86, 056601 (2012).

[5] See, e.g., A. Montina, U. Bortolozzo, S. Residori, and F. T. Arecchi, Phys. Rev. Lett. 103, 173901 (2009); J. Kasparian, P. Béjot, J. P. Wolf, and J. M. Dudley, 
Opt. Express 17, 12070 (2009); C. Bonatto, Michael Feyereisen, S. Barland, M. Giudici, C. Masoller, J. R. Rios Leite, and J. R. Tredicce, Phys. Rev. Lett. 107, 053901 (2011); J. ZamoraMunt, B. Garbin, S. Barland, M. Giudici, J. R. Rios Leite, C. Masoller, and J. R. Tredicce, Phys. Rev. A 87, 035802 (2013); K. Hammani, A. Picozzi, and C. Finot, Opt. Commun. 284, 2594 (2011); C. Lecaplain, P. Grelu, J. M. Soto-Crespo, and N. Akhmediev, Phys. Rev. Lett. 108, 233901 (2012); D. V. Churkin, O. A. Gorbunov, and S. V. Smirnov, Opt. Lett. 36, 3617 (2011); A. N. Pisarchik, R. Jaimes-Reátegui, R. Sevilla-Escoboza, G. Huerta-Cuellar, and M. Taki, Phys. Rev. Lett. 107, 274101 (2011); M. I. Kolobov, A. Mussot, A. Kudlinski, E. Louvergneaux, and M. Taki, Phys. Rev. A 83, 035801 (2011); D. Majus, V. Jukna, G. Valiulis, D. Faccio, and A. Dubietis, ibid. 83, 025802 (2011); F. Baronio, A. Degasperis, M. Conforti, and S. Wabnitz, Phys. Rev. Lett. 109, 044102 (2012); E. Louvergneaux, V. Odent, M. I. Kolobov, and M. Taki, Phys. Rev. A 87, 063802 (2013); F. Baronio, A. Degasperis, M. Conforti, and S. Lombardo, Phys. Rev. Lett. 111, 114101 (2013); S. Birkholz, E. T. J. Nibbering, C. Brée, S. Skupin, A. Demircan, G. Genty, and G. Steinmeyer, ibid. 111, 243903 (2013); F. Baronio, M. Conforti, A. Degasperis, S. Lombardo, M. Onorato, and S. Wabnitz, ibid. 113, 034101 (2014); A. K. Dal Bosco, D. Wolfersberger, and M. Sciamanna, Opt. Lett. 38, 703 (2013); N. Marsal, V. Caullet, D. Wolfersberger, and M. Sciamanna, ibid. 39, 3690 (2014); T. Roger, D. Majus, G. Tamosauskas, P. Panagiotopoulos, M. Kolesik, G. Genty, I. Gražulevičiūte, A. Dubietis, and D. Faccio, Phys. Rev. A 90, 033816 (2014).

[6] A. F. J. Runge, C. Aguergaray, N. G. R. Broderick, and M. Erkintalo, Opt. Lett. 38, 4327 (2013).

[7] M. Shats, H. Punzmann, and H. Xia, Phys. Rev. Lett. 104, 104503 (2010).

[8] A. N. Ganshin, V. B. Efimov, G. V. Kolmakov, L. P. MezhovDeglin, and P. V. E. McClintock, Phys. Rev. Lett. 101, 065303 (2008).

[9] R. Höhmann, U. Kuhl, H. J. Stöckmann, L. Kaplan, and E. J. Heller, Phys. Rev. Lett. 104, 093901 (2010).

[10] N. Akhmediev, J. M. Soto-Crespo, and A. Ankiewicz, Phys. Lett. A 373, 2137 (2009).

[11] K. L. Henderson, D. H. Peregrine, and J. W. Dold, Wave Motion 29, 341 (1999); N. Akhmediev, A. Ankiewicz, and J. M. SotoCrespo, Phys. Rev. E 80, 026601 (2009); A. Ankiewicz, J. M. Soto-Crespo, and N. Akhmediev, ibid. 81, 046602 (2010); S. Randoux, P. Walczak, M. Onorato, and P. Suret, Phys. Rev. Lett. 113, 113902 (2014).

[12] D. H. Peregrine, J. Australian Math. Soc., Ser. B. Appl. Math. 25, 16 (1983); K. Hammani et al., Opt. Lett. 36, 112 (2011); B. Kibler, J. Fatome, C. Finot, G. Millot, G. Genty, B. Wetzel, N. Akhmediev, F. Dias, and J. M. Dudley, Sci. Rep. 2, 463 (2012); B. Frisquet, B. Kibler, and G. Millot, Phys. Rev. X 3, 041032 (2013); B. Frisquet, A. Chabchoub, J. Fatome, C. Finot, B. Kibler, and G. Millot, Phys. Rev. A 89, 023821 (2014).

[13] N. Akhmediev and E. Pelinovsky, Editorial-Introductory remarks on Discussion \& Debate: Rogue Waves-Towards a Unifying Concept, Eur. Phys. J. Spec. Top. 185, 1 (2010).

[14] M. Onorato et al., Phys. Rep. 528, 47 (2013).

[15] N. Akhmediev, J. M. Dudley, D. R. Solli, and S. K. Turitsyn, J. Opt. 15, 060201 (2013).

[16] J. M. Dudley, F. Dias, M. Erkintalo, and G. Genty, Nat. Photon. 8, 755 (2014).
[17] M. Onorato, A. R. Osborne, M. Serio, L. Cavaleri, C. Brandini, and C. T. Stansberg, Phys. Rev. E 70, 067302 (2004).

[18] C. Viotti, D. Dutykh, J. M. Dudley, and F. Dias, Phys. Rev. E 87, 063001 (2013).

[19] A. Picozzi et al., Phys. Rep. 542, 1 (2014).

[20] J. Laurie, U. Bortolozzo, S. Nazarenko, and S. Residori, Phys. Rep. 514, 121 (2012).

[21] K. Hammani, B. Kibler, C. Finot, and A. Picozzi, Phys. Lett. A 374, 3585 (2010).

[22] B. Kibler, K. Hammani, C. Finot, and A. Picozzi, Phys. Lett. A 375, 3149 (2011).

[23] V. E. Zakharov, F. Dias, and A. Pushkarev, Phys. Rep. 398, 1 (2004).

[24] V. E. Zakharov and E. A. Kuznetsov, J. Exp. Theor. Phys. 86, 1035 (1998).

[25] B. Rumpf, A. C. Newell, and V. E. Zakharov, Phys. Rev. Lett. 103, 074502 (2009).

[26] N. Akhmediev and M. Karlsson, Phys. Rev. A 51, 2602 (1995).

[27] D. V. Skryabin and A. V. Gorbach, Rev. Mod. Phys. 82, 1287 (2010).

[28] V. E. Zakharov, V. S. L'vov, and G. Falkovich, Kolmogorov Spectra of Turbulence I (Springer, Berlin, 1992).

[29] S. Nazarenko, Wave Turbulence, Lectures Notes in Physics 825 (Springer, Berlin, 2011).

[30] C. Michel et al., Opt. Lett. 35, 2367 (2010); P. Suret, S. Randoux, H. R. Jauslin, and A. Picozzi, Phys. Rev. Lett. 104, 054101 (2010).

[31] B. Rumpf and A. C. Newell, Phys. Rev. Lett. 87, 054102 (2001); B. Rumpf, Phys. Rev. E 77, 036606 (2008).

[32] C. Connaughton, C. Josserand, A. Picozzi, Y. Pomeau, and S. Rica, Phys. Rev. Lett. 95, 263901 (2005); G. Düring, A. Picozzi, and S. Rica, Phys. D (Amsterdam) 238, 1524 (2009); A. Picozzi and S. Rica, Opt. Commun. 285, 5440 (2012).

[33] P. Aschieri, J. Garnier, C. Michel, V. Doya, and A. Picozzi, Phys. Rev. A 83, 033838 (2011).

[34] S. Babin, D. Churkin, A. Ismagulov, S. Kablukov, and E. Podivilov, J. Opt. Soc. Am. B 24, 1729 (2007).

[35] E. G. Turitsyna, G. Falkovich, V. K. Mezentsev, and S. K. Turitsyn, Phys. Rev. A 80, 031804 (2009); E. G. Turitsyna, G. Falkovich, A. El-Taher, X. Shu, P. Harper, and S. K. Turitsyn, Proc. R. Soc. London, Ser. A 468, 2496 (2012).

[36] E. G. Turitsyna et al., Nat. Photon. 7, 783 (2013).

[37] R. Weill, B. Fischer, and O. Gat, Phys. Rev. Lett. 104, 173901 (2010); R. Weill, B. Levit, A. Bekker, O. Gat, and B. Fischer, Opt. Express 18, 16520 (2010); G. Oren, A. Bekker, and B. Fischer, Optica 1, 145 (2014).

[38] C. Conti, M. Leonetti, A. Fratalocchi, L. Angelani, and G. Ruocco, Phys. Rev. Lett. 101, 143901 (2008).

[39] I. Carusotto and C. Ciuti, Rev. Mod. Phys. 85, 299 (2013).

[40] J. Klaers, J. Schmitt, F. Vewinger, and M. Weitz, Nature (London) 468, 545 (2010).

[41] C. Michel, M. Haelterman, P. Suret, S. Randoux, R. Kaiser, and A. Picozzi, Phys. Rev. A 84, 033848 (2011).

[42] L. A. Lugiato and R. Lefever, Phys. Rev. Lett. 58, 2209 (1987).

[43] T. Arecchi, S. Boccalettic, and P. Ramazza, Phys. Rep. 318, 1 (1999).

[44] F. Leo et al., Nat. Photon. 4, 471 (2010).

[45] F. Leo, A. Mussot, P. Kockaert, P. Emplit, M. Haelterman, and M. Taki, Phys. Rev. Lett. 110, 104103 (2013). 
[46] J. K. Jang, M. Erkintalo, S. G. Murdoch, and S. Coen, Nat. Photon. 7, 657 (2013).

[47] G. P. Agrawal, Nonlinear Fiber Optics, 5th ed. (Academic, New York, 2012).

[48] T. Carmon, M. Soljacic, and M. Segev, Phys. Rev. Lett. 89, 183902 (2002); H. Buljan, M. Soljačić, T. Carmon, and M. Segev, Phys. Rev. E 68, 016616 (2003).

[49] B. Barviau et al., Opt. Express 17, 7392 (2009); B. Barviau, B. Kibler, and A. Picozzi, Phys. Rev. A 79, 063840 (2009); B. Kibler, C. Michel, A. Kudlinski, B. Barviau, G. Millot, and A. Picozzi, Phys. Rev. E 84, 066605 (2011); B. Barviau, J. Garnier, G. Xu, B. Kibler, G. Millot, and A. Picozzi, Phys. Rev. A 87, 035803 (2013).
[50] A. Sauter et al., Opt. Lett. 30, 2143 (2005).

[51] O. A. Gorbunov, S. Sugavanam, and D. V. Churkin, Opt. Express 22, 28071 (2014).

[52] L. Pitaevskii and S. Stringari, Bose-Einstein Condensation (Oxford Science, Oxford, 2003).

[53] A. Picozzi and J. Garnier, Phys. Rev. Lett. 107, 233901 (2011).

[54] J. Garnier and A. Picozzi, Phys. Rev. A 81, 033831 (2010); J. Garnier, G. Xu, S. Trillo, and A. Picozzi, Phys. Rev. Lett. 111, 113902 (2013); B. Kibler, C. Michel, J. Garnier, and A. Picozzi, Opt. Lett. 37, 2472 (2012); G. Xu et al., ibid. 39, 4192 (2014); Phys. Rev. A 90, 013828 (2014). 